\title{
Metastatic Malignant Neoplasm in the Sellar Region
}

National Cancer Institute

\section{Source}

National Cancer Institute. Metastatic Malignant Neoplasm in the Sellar Region. NCI

Thesaurus. Code C155786.

A malignant neoplasm that has metastasized to the sellar region from another anatomic site. 